\title{
Chemical Constituents from the Branches of Carpinus turczaninowii with Antioxidative Activities
}

\author{
Ha Na Ko, Jung Mi Kim, Hee Jung Bu, and Nam Ho Lee* \\ Department of Chemistry and Cosmetic Science Research Center, Jeju National University, \\ Ara-1, Jeju 690-756, Korea. 'E-mail: namho@jejunu.ac.kr
}

(Received June 11, 2013; Accepted July 15, 2013)

Key words: Carpinus turczaninowii, DPPH free radical scavenger, $\mathrm{ABTS}^{+}$radical, Isolation, Structure determination

Korean hornbeam Carpinus turczaninowii is a deciduous woody plant belonging to the family Betulaceae. This flora is endemic to Korea, and can reach a height of $15 \mathrm{~m}$. In the autumn, the fallen leaves of $C$. turczaninowii display a beautiful orange-red color and the tree is commonly used for bonsai in Korea. The wood is very hard, dense and fine textured, and has been used for making agricultural tools and furniture. Previous chemical investigation on this plant indicated only the existence of flavonoids such as naringenin and quercetin glycosides from the leaves. ${ }^{1}$ We have recently reported the isolation diarylheptanoids possessing anti-inflammatory activities from the ethanol extract of C. turczaninowii. $^{2}$

Reactive oxygen species (ROS) are chemically reactive substances which can attack biomolecules, lipids, proteins and nucleic acid within living organisms. ROS can be classified into two groups: oxygen-centered free radicals $\left(\mathrm{O}_{2}{ }^{-}, \mathrm{HO} \cdot \mathrm{RO} \cdot\right.$ and $\left.\mathrm{ROO} \cdot\right)$, and non-radical molecules $\left({ }^{1} \mathrm{O}_{2}\right.$ and $\left.\mathrm{H}_{2} \mathrm{O}_{2}\right)$. It is known that the formation of ROS is the normal metabolic processes. ${ }^{3}$ However, produced in excess or in certain physiological conditions, the generated ROS can cause destructive and harmful effects to human tissues, causing various diseases which are mainly associated with aging. ${ }^{4}$ Humans have evolved defense systems which employ antioxidative enzymes to prevent detrimental oxidation. Along with this endogenous protective mechanism, it is believed that exogenous antioxidants are necessary to decrease the cumulative effects of ROS-related damage. Important antioxidants in diet include vitamins and flavonoids from plant sources. Antioxidants are also utilized in the food and cosmetic industries in order to protect from damages associated with ROS. Due to their beneficial effects in broad areas, novel antioxidative agents especially from natural sources have attracted much attention in terms of their ecologically friendly properties. ${ }^{5}$

We are continuously conducting research projects to find biologically active natural products from plants growing in Jeju Island, a place of great biodiversity located at the southernmost part of Korea. ${ }^{6}$ During our investigation, we observed that the ethanol extract prepared from the branches of C. turczaninowii exhibited significant DPPH radical scavenging activities. Therefore, we decided to carry out a full phytochemical investigation of the extract to determine the bioactive metabolites. We herein described the isolation and structure determination of the chemical constituents. In addition, antioxidative properties for the isolates were examined using the $\mathrm{DPPH}$ and $\mathrm{ABTS}^{+}$radical scavenging activity tests.

The plant extract (78.1 g) was prepared using 70\% ethanol from the branches of $C$. turczaninowii $(650.0 \mathrm{~g})$ at room temperature, with a $12.0 \%$ yield. It was suspended in water and partitioned successively into $n$-hexane, ethyl acetate (EtOAc), and $n$-butanol. All extracts were then tested for their DPPH radical scavenging activities, which are shown in Fig. 1. Ascorbic acid ( $\mathrm{SC}_{50}, 50 \%$ scavenging concentration, $10.3 \mu \mathrm{g} / \mathrm{mL}$ ) was used as a positive control in this experiment. The ethanol extract exhibited considerable activity showing an $\mathrm{SC}_{50}$ value of $28.8 \mu \mathrm{g} / \mathrm{mL}$. Among the solvent fractions, the EtOAc soluble one revealed the highest potency with $\mathrm{SC}_{50} 13.7 \mu \mathrm{g} / \mathrm{mL}$. Its activity was comparable to the positive control, ascorbic acid.

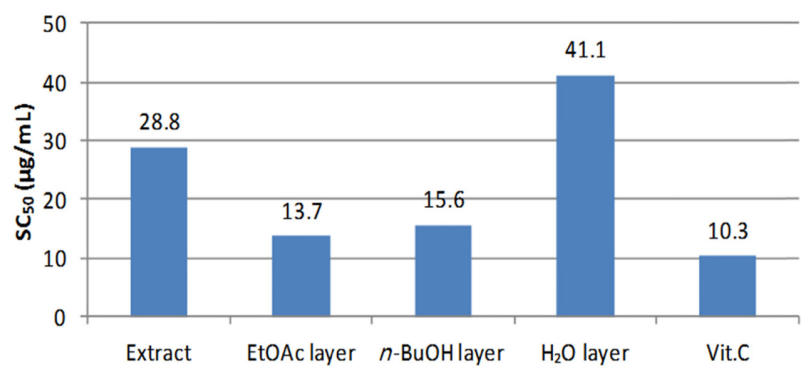

Figure 1. DPPH radical scavenging activities of the solvent fractions from C. turczaninowii. 


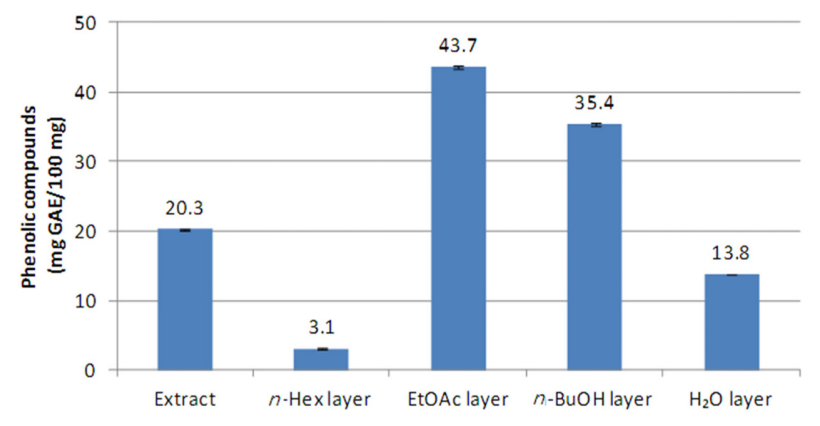

Figure 2. Total polyphenol contents for the fractions from $C$. turczaninowii.

Polyphenols are chemicals which are characterized by the presence of large multiples of phenol structural units. The most abundant polyphenols are tannins and flavonoids, which are found in virtually all families of plants. It is well established that polyphenol compounds function as natural antioxidants by scavenging radical species. As the solvent fractions from C. turczaninowii exhibited DPPH radical inhibition activities, the total polyphenolic contents were measured for their extract and fractions (Fig. 2). Quantification of total phenols was based on colorimetric measurement using the Folin-Ciocalteu reaction. ${ }^{7}$ The gallic acid equivalent (GAE) per $100 \mathrm{mg}$ sample was calculated based on the calibration curve. As shown in Fig. 2, the ethanol extract was found to have $20.3 \mathrm{mg}$ GAE polyphenols. Comparing the solvent fractions, the EtOAc fraction exhibited the highest polyphenol concentration, with 43.7 mg GAE. Since the EtOAc fraction showed potent radical scavenging activities and higher contents of polyphenols, it was chosen for further fractionation to isolate the active constituents.

The EtOAc-soluble layer was subjected to vacuum liquid chromatography with gradients of $n$-hexane/EtOAc followed by EtOAc/methanol to provide 30 fractions. Each fraction was purified by repeated column chromatography with silica gel or Sephadex LH-20, leading to the isolation of eight compounds (1-8) (Fig. 3). The characterizations of the chemical structures for the isolates were accomplished by a spectroscopic method, including $1 \mathrm{D}$ and $2 \mathrm{D}$ NMR data. The structures of the known compounds (1-8) were further confirmed by comparison of their spectroscopic data to literature values.

Compound $\mathbf{1}$ showed 29 signals in its ${ }^{13} \mathrm{C}$ NMR spectrum, where two olefinic carbons and one oxygen-bearing carbon were easily identified. The presence of six methyl groups were also suggested based on ${ }^{1} \mathrm{H}$ and DEPT NMR signals. Based on the other spectroscopic data, compound $\mathbf{1}$ was
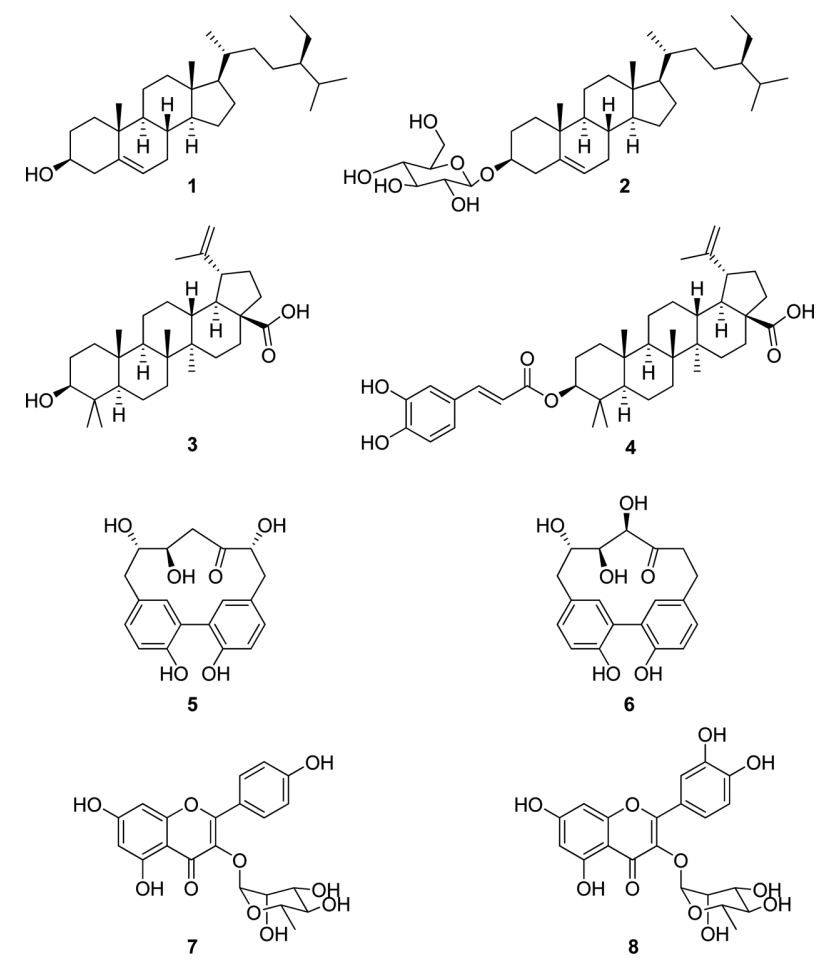

Figure 3. Structures of compounds isolated from C. turczaninowii.

verified as the sterol compound, $\beta$-sitosterol. ${ }^{8}$ Compared to sterol $\mathbf{1}$, compound $\mathbf{2}$ displayed a similar ${ }^{13} \mathrm{C}$ NMR pattern, except for the presence of an additional six oxygenbearing carbon signals. Investigation of chemical shifts of the carbon signals suggested the attachment of a glucose unit in 2. The large coupling constant for the anomeric proton $(\delta 5.07, J=7.6 \mathrm{~Hz})$ indicated its $\beta$-configuration. Therefore, compound 2 was identified as sitosterol $3-O-\beta$ D-glucopyranoside (daucosterol). ${ }^{9}$ Compound $\mathbf{3}$ displayed 30 signals, including six methyl carbons in the DEPT NMR spectrum, which suppose its triterpene skeleton. One carboxylic $(\delta 180.2)$ and vinyl carbons ( $\delta 152.1$ and 110.3) were also observed in the ${ }^{13} \mathrm{C}$ spectrum. Investigation of these data along with the proton spectrum suggests compound 3 to be betulinic acid, which was confirmed by comparison with literature data. ${ }^{10}$ Compound $\mathbf{4}$ was also inferred as an analogue of betulinic acid based on ${ }^{1} \mathrm{H}$ and ${ }^{13} \mathrm{C}$ NMR data. It was surmised that an aromatic ester bearing an olefin was attached to the $\mathrm{C}-3$ position. The trans-configured olefin was verified by proton signals at $\mathrm{d}$ $6.22(1 \mathrm{H}, \mathrm{d}, J=15.9 \mathrm{~Hz})$ and $7.43(1 \mathrm{H}, J=15.9 \mathrm{~Hz})$. Proton NMR data indicated the existence of a 1,3,5-trisubstituted benzene ring. Therefore, it was determined that the caffeic acid moiety was attached via an ester bond to the hydroxyl at C-3 in betulinic acid. By comparing with 
literature data, compound $\mathbf{4}$ was confirmed as betulinic acid $3 \beta$ - $O$-caffeate (pyracrenic acid). ${ }^{11}$ Compounds 5 and 6 were identified as carpinontriol B and carpinontriol A respectively, and were previously isolated from C. turczaninowi. ${ }^{2}$ These compounds, bearing diarylheptanoid skeletons, were reported to have anti-inflammatory activities by inhibition of nitric oxide production. Investigation of the ${ }^{1} \mathrm{H}$ NMR spectrum indicated that compound 7 has an aromatic ring possessing meta-coupled protons showing peaks at $\delta 6.18(1 \mathrm{H}, \mathrm{d}, J=2.1 \mathrm{~Hz})$ and $6.35(1 \mathrm{H}$, $\mathrm{d}, J=2.1 \mathrm{~Hz}$ ). An additional symmetric aromatic ring was also recognized by the presence of peaks and ortho-coupling constants at $\delta 6.92(2 \mathrm{H}, \mathrm{d}, J=9.0 \mathrm{~Hz})$ and $7.78(2 \mathrm{H}$, $\mathrm{d}, J=9.0 \mathrm{~Hz})$. The ${ }^{13} \mathrm{C}$ NMR spectrum also revealed a carbonyl ( $\delta 179.5)$ and olefinic carbons ( $\delta 136.2$ and 158.6). These data suggest compound 7 to have a skeleton of a flavonol, kaempferol. Besides the flavonoid frame, a sugar rhamnosyl residue, as indicated by the double methyl at $\delta 0.92$, was determined to be attached at the C-3 position. Therefore, the compound 7 was identified as kaempferol 3-O- $\alpha$-rhamnopyranoside, afzelin. ${ }^{12}$ Compound 8 exhibited an almost identical NMR spectra to compound 7 differing only in the presence of a 1,3,4-trisubstituted aromatic ring, instead of a symmetric 1,4-disubstitued one in the flavonoid 7. Therefore, compound $\mathbf{8}$ was identified as quercetin $3-O$ - $\alpha$-rhamnopyronoside, quercitrin. ${ }^{13}$

The antioxidative activities were examined employing a DPPH free radical scavenge method. ${ }^{14}$ DPPH (2,2-diphenyl1-picrylhydrazyl) is a relatively stable radical species bearing a characteristic deep violet color in solution, and radical scavenging activity can be determined by a loss of DPPH absorbance at $515 \mathrm{~nm}$. The inhibition activities of the isolated compounds were measured at concentrations of 3.0$100 \mu \mathrm{g} / \mathrm{mL}$, and the results are summarized in Table 1 . This activity was expressed as $\mathrm{SC}_{50}$, the concentration needed to reduce $50 \%$ of DPPH. Ascorbic acid was used as a positive control, having an $\mathrm{SC}_{50}$ value of $43.5 \mu \mathrm{M}$.
Among the isolates, pyracrenic acid (4) and quercitrin (8) showed the highest free radical scavenging activities, with $\mathrm{SC}_{50}$ values of 55.2 and $62.4 \mu \mathrm{M}$, respectively, comparable to that of the positive control. The diarylheptanoids ( 5 and 6) showed some activity, with $\mathrm{SC}_{50}$ values of 244.3 , $360.5 \mu \mathrm{M}$, respectively. As another method to determine in vitro anti-oxidation capacities for the extracts and isolates, an ABTS [2,2'-azino-bis(3-ethylbenzothiazoline6-sulfonic acid)] assay was employed..$^{15}$ ABTS is a chemical which can be oxidized by persulfate to yield an ABTS radical cation $\left(\mathrm{ABTS}^{+}\right)$featuring a characteristic blue green color in solution. In the presence of antioxidants such as phenolics, this radical cation is converted back to its colorless neutral form, which can be monitored spectrophotometrically. Upon subjection of this assay to the ethanol extract and solvent fractions, EtOAc and $n$-butanol fractions exhibited very strong radical scavenging activities (Fig. 4). As in the DPPH experiment, ascorbic acid was used the positive control. It is interesting to note that EtOAc and $n$-butanol fractions showed more potent activities than ascorbic acid. This ABTS assay was conducted for the isolated compounds. As shown in Table 1, quercitrin (8) showed the most potent radical scavenging activities among the isolates, with an $\mathrm{SC}_{50}$ value $29.6 \mu \mathrm{M}$, which demonstrates that it is more

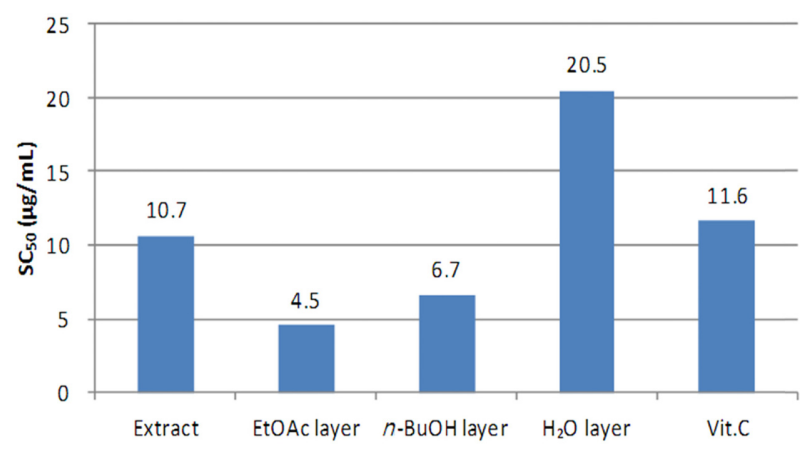

Figure 4. $\mathrm{ABTS}^{+}$radical scavenging activities of the solvent fractions from $C$. turczaninowii.

Table 1. $\mathrm{SC}_{50}$ values of $\mathrm{DPPH}$ and $\mathrm{ABTS}^{+}$radical scavenging activities of the isolated compounds 1-8 from C. turczaninowi

\begin{tabular}{cccc}
\hline Compound No. & Compound name & $\mathrm{DPPH} \mathrm{SC}_{50}(\mu \mathrm{M})$ & $\mathrm{ABTS}^{+} \mathrm{SC}_{50}(\mu \mathrm{M})$ \\
\hline $\mathbf{1}$ & $\beta$-sitosterol & $>500$ & $>100$ \\
$\mathbf{2}$ & daucosterol & $>500$ & $>100$ \\
$\mathbf{3}$ & betulinic acid & $>500$ & 3100 \\
$\mathbf{4}$ & pyracrenic acid & 55.2 & 42.1 \\
$\mathbf{5}$ & carpinontriol B & 244.3 & 45.8 \\
$\mathbf{6}$ & carpinontriol A & 360.5 & $>100$ \\
$\mathbf{7}$ & afzelin & $>500$ & 29.6 \\
$\mathbf{8}$ & quercitrin & 62.4 & 31.6 \\
\hline
\end{tabular}


active than the positive control. Compounds 4-6 also showed very strong antioxidative activities which were comparable to that of ascorbic acid (Table 1).

As expected from its catechol structure, quercitrin (8) displayed the most outstanding radical scavenging activities. Its protective effects against different types of oxidative stress have been studied in vitro as well as in vivo models. ${ }^{16}$ Pyracrenic acid (4) has been reported to have strong DPPH radical scavenging activities. ${ }^{17}$ The catechol group in its caffeate moiety could be responsible for the inhibitory activities on DPPH and $\mathrm{ABTS}^{+}$radicals. As shown in Table 1, capinontriols exhibited potent $\mathrm{ABTS}^{+}$radical scavenging activities, on which no prior report has been disclosed yet.

In conclusion, eight compounds (1-8) were identified in ethanol extracts prepared from the branches of $C$. turczaninowii. The compounds, besides $\mathbf{5}$ and $\mathbf{6}$, were isolated for the first time from this woody plant. Pyracrenic acid (4) and quercitrin (8) showed potent DPPH free radical scavenging activities with $\mathrm{SC}_{50}$ values of 55.2 and $62.4 \mu \mathrm{M}$, respectively, where ascorbic acid $\left(\mathrm{SC}_{50} 43.5 \mu \mathrm{M}\right)$ was used as a positive control. Compounds 4, 5, 6 and 8 showed strong activities in $\mathrm{ABTS}^{+}$radical scavenging assay, with $\mathrm{SC}_{50}$ values of 34.1, 42.1, 45.8 and $29.6 \mu \mathrm{M}$, respectively. These activities are comparable in potency to ascorbic acid $\left(\mathrm{SC}_{50} 31.6 \mu \mathrm{M}\right)$. Based on these results, C. turczaninowii extracts are expected to be useful antioxidative agents, potentially applicable in food or cosmetic industries, based on the results of further studies.

\section{EXPERIMENTAL}

\section{Reagents and Instruments}

DPPH (2,2-diphenyl-1-picrylhydrazyl) and ABTS [2,2'azino-bis(3-ethylbenzothiazoline-6-sulfonic acid)] were purchased from Aldrich. All solvents used were of analytical grade. A Biochrom Libra S22 UV-visible spectrophotometer was used for the screening of radical inhibition activities. ${ }^{1} \mathrm{H}$ (400 MHz) and ${ }^{13} \mathrm{C}(100.6 \mathrm{MHz})$ nuclear magnetic resonance (NMR) spectra were recorded on JNM-LA 400 instrument (JEOL) with chemical shift $(\delta)$ data were reported in ppm relative to the solvent used. Merck silica gel (0.063$0.2 \mathrm{~mm}$ ) was used for normal phased column chromatography. Silica gel $60 \mathrm{~F}_{254}$ coated on aluminum plates by Merck were used for thin layer chromatography (TLC). Gel filtration chromatography (GFC) was performed using Sephadex LH-20 (25-100 $\mu \mathrm{m})$ from Fluka.

\section{Plant Material}

The branches of Carpinus turczaninowii were collected in December 2009 from Halla Botanical Garden in Jeju Island, Korea. Voucher specimen (sample number 308) was deposited at the herbarium of Department of Chemistry, Jeju National University.

\section{Extraction and Isolation}

The shade dried C. turczaninowii $(650.0 \mathrm{~g})$ was cut into small pieces, and extracted with $70 \%$ ethanol (14 L) two times at room temperature for $24 \mathrm{~h}$. The gummy extract $(78.1 \mathrm{~g})$ was obtained after concentration of the filtered solution. Part of the ethanol extract (26.5 g) was suspended in water $(2 \mathrm{~L})$, and fractionated into $n$-hexane $(0.30 \mathrm{~g})$, ethyl acetate $(3.8 \mathrm{~g}), n$-butanol $(7.0 \mathrm{~g})$ and water $(16.1 \mathrm{~g})$ portions. The ethyl acetate (EtOAc) layer was subjected to vacuum liquid chromatography (VLC) on silica gel, using step-gradients ( $n$-hexane/EtOAc to EtOAc/MeOH, $300 \mathrm{~mL}$ each) to provide 30 fractions (V1-V30). The compounds $\mathbf{1}$ (13.3 mg), 3 (24.3 mg) and $\mathbf{4}(37.4 \mathrm{mg}$ ) were obtained from fractions V4, V5 and V8 respectively by recrystallization. Fraction V13 was purified by silica gel column chromatography (CC) using chloroform-EtOAc-methanol (2:3:1) to afford the compound $5(26.9 \mathrm{mg})$. The fractions V17 and V18 were combined, and purified by silica gel CC with chloroform-EtOAc-methanol (3:3:1) to give compound $6(36.0 \mathrm{mg})$. The fraction V19 was subjected to silica gel $\mathrm{CC}$ with chloroform-EtOAc-methanol (3:3:1) followed by Sephadex LH-20 CC chloroform-EtOAc-methanol (3:2:1) to isolate the compounds $7(6.1 \mathrm{mg})$ and $8(8.9 \mathrm{mg})$. The compound $2(8.1 \mathrm{mg})$ was isolated from the fraction V21 as a methanol-insoluble substance.

Acknowledgments. This research was supported by the 2013 scientific promotion program, funded by Jeju National University.

\section{REFERENCES}

1. Jeon, J. I.; Jang, J. S. Korean J. Plant Tax. 2000, 30, 139.

2. Ko, H. N.; Oh, T. H.; Baik, J. S.; Hyun, C. G.; Kim, S. S.; Lee, N. H. Int. J. Pharmacol. 2013, 9, 157.

3. Kowaltowski, A. J.; Souza-Pinto, N. C.; Castilho, R. F.; Vercesi, A. E. Free Radical Biol. Med. 2009, 47, 333.

4. Liochev, S. I. Free Radical Biol. Med. 2013, 60, 1.

5. Moure, A.; Cruz, J. M.; Franco, D.; Dominguez, J. M.; Sineiro, J.; Dominguez, H.; Nunez, M. J.; Parajo, J. C. Food Chem. 2001, 72, 145.

6. (a) Kim, J. H.; Kim, J. E.; Bu, H. J.; Lee, N. H. Bull. Korean Chem. Soc. 2012, 33, 344. (b) Park, S. H.; Oh, T. H.; Kim, S. S.; Kim, J. E.; Lee, S. J.; Lee, N. H. J. Enzym. Inhib. Med. Chem. 2012, 27, 390. (c) Ko, R. K.; Kang, M. C.; Kim, S. S.; Oh, T. H.; Kim, G. O.; Hyun, C. G.; 
Hyun, J. W.; Lee, N. H. Nat. Prod. Commun. 2013, 8, 427.

7. Folin, O.; Denis, W. J. Biol. Chem. 1915, 22, 305.

8. Sohn, B. H.; Park, J. H.; Lee, D. Y.; Cho, J. G.; Kim, Y. S.; Jung, I. S.; Kang, P. D.; Baek, N. I. J. Korean Soc. Appl. Biol. Chem. 2009, 52, 336.

9. Lee, S. H.; Kim, K. S.; Jang, J. M.; Park, Y. M.; Kim, Y. B.; Kim, B. K. Arch. Pharm. Res. 2002, 25, 285.

10. Yun, Y. H.; Han, S. H.; Park, E. J.; Yim, D. S.; Lee, S. K.; Lee, C. K.; Cho, K. H.; Kim, K. J. Arch. Pharm. Res. 2003, 26, 1087.

11. Kim, J. H.; Byun, J. C.; Bandi, A. K. R.; Hyun, C. G.; Lee, N. H. J. Med. Plants Res. 2009, 3, 914.

12. Chung, S. K.; Kim, Y. C.; Takaya, Y.; Terachima, K.; Niwa,
M. J. Agric. Food Chem. 2004, 54, 4664.

13. Lee, J. H.; Ku, C. H.; Baek, N. I.; Kim, S. H.; Park, H. W.; Kim, D. K. Arch. Pharm. Res. 2004, 27, 40.

14. Blois, M. S. Nature 1958, 181, 1199.

15. Re, R.; Pellegrini, N.; Proteggente, A.; Pannala, A.; Yang, M.; Rice-Evans, C. Free Radical Biol. Med. 1999, 26, 1231.

16. Yang, H. M.; Ham, Y. M.; Yoon, W. J.; Roh, S. W.; Jeon, Y. J.; Oda, T.; Kang, S. M.; Kang, C. M.; Kim, E. A.; Kim, D. K.; Kim, K. N. J. Photochem. Photobiol. B-Biol. 2012, 114, 126.

17. Kim, J. H.; Byun, J. C.; Bandi, A. K. R.; Hyun, C. G.; Lee, N. H. J. Med. Plants Res. 2009, 3, 914. 